Document downloaded from:

http://hdl.handle.net/10251/79774

This paper must be cited as:

Defant, A.; Sevilla Peris, P. (2012). Convergence of monomial expansions in banach spaces. Quarterly Journal of Mathematics. 63(3):569-584. doi::10.1093/qmath/haq053.

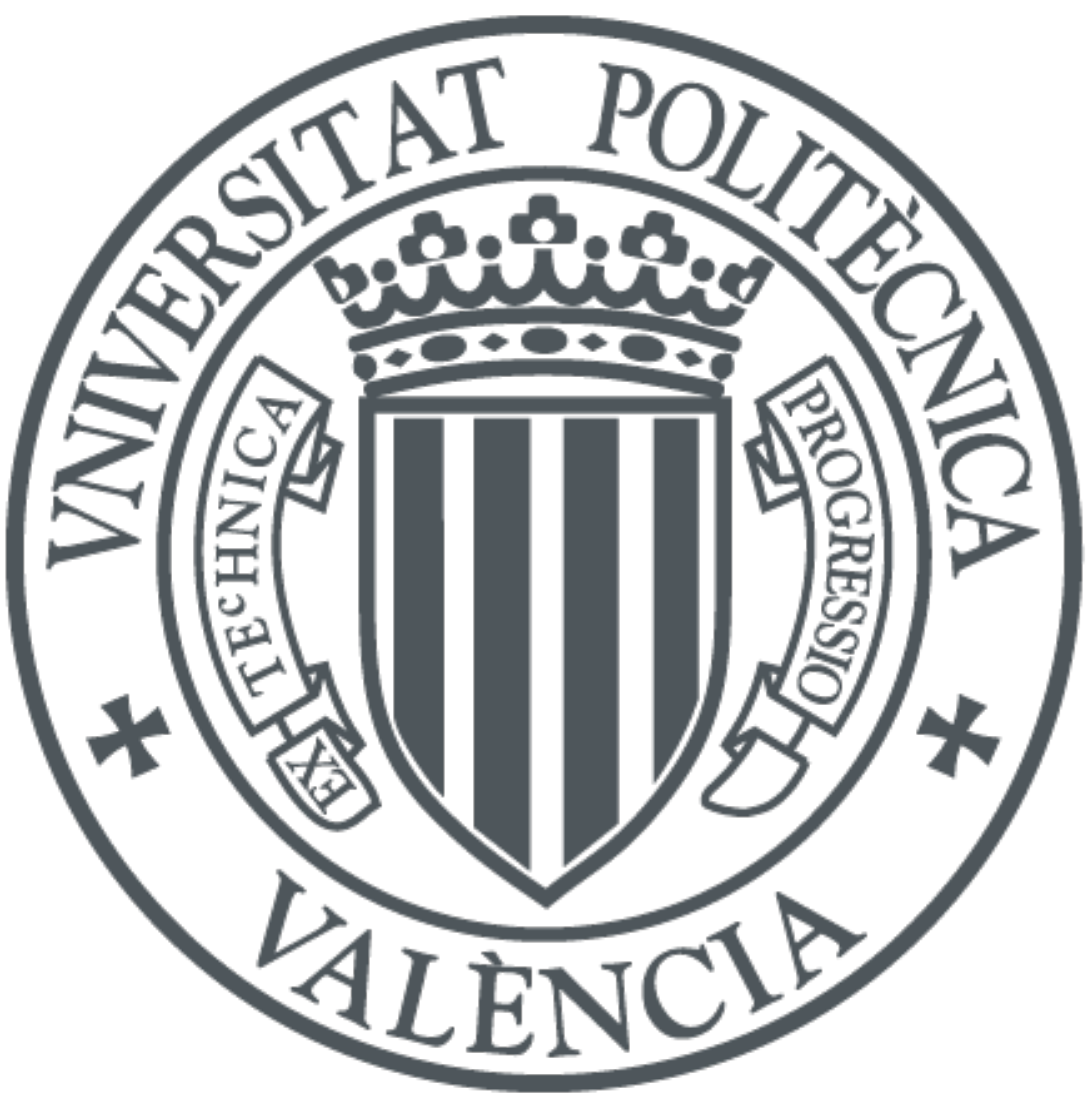

The final publication is available at

http://dx.doi.org/10.1093/qmath/haq053

Copyright Oxford University Press (OUP)

Additional Information 


\title{
CONVERGENCE OF MONOMIAL EXPANSIONS IN BANACH SPACES
}

\author{
ANDREAS DEFANT AND PABLO SEVILLA-PERIS
}

\begin{abstract}
If $E$ is a Banach sequence space, then each holomorphic function defines a formal power series $\sum_{\alpha} c_{\alpha}(f) z^{\alpha}$. The problem of when such an expansion converges absolutely and actually represents the function goes back to the very beginning of the theory of holomorphic functions on infite dimensional spaces. Several very deep results have been given for scalar valued functions by Ryan, Lempert and Defant, Maestre and Prengel. We go on with this study, looking at monomial expansions of vector valued holomorphic functions on Banach spaces. Some situations are very different from the scalar valued case.
\end{abstract}

\section{INTRODUCTION, MAIN RESULTS AND PRELIMINARIES}

If $\left(E_{n},\|\|\right)$ is a finite dimensional Banach space and $Y$ is any Banach space it is a well known fact that every holomorphic (i.e. complex Fréchet differentiable) function $f: U \rightarrow Y\left(U \subseteq E_{n}\right.$ open and containing 0$)$ has a power series expansion

$$
f(z)=\sum_{\alpha \in \mathbb{N}_{0}^{n}} c_{\alpha}(f) z^{\alpha}
$$

where $z^{\alpha}=z_{1}^{\alpha_{1}} \cdots z_{n}^{\alpha_{n}}$ and the coefficients $c_{\alpha}(f) \in Y$ can be calculated either via the Cauchy integral formula or partial derivations (see e.g. [12, Section 3.1] or [8]). Our aim in this paper is to study the situation in the infinite dimensional setting. We consider Banach sequence spaces (often also called Köthe sequence spaces) i.e. Banach spaces $E \subseteq \mathbb{C}^{\mathbb{N}}$ of sequences such that $\ell_{1} \subseteq E \subseteq \ell_{\infty}$ satisfying that if $x \in \mathbb{C}^{\mathbb{N}}$ and $y \in E$ are so that $\left|x_{n}\right| \leq\left|y_{n}\right|$ for every $n$ then $x \in E$ and $\|x\| \leq\|y\|$. We denote by $e_{n}$ the $n$-th canonical unit vector $\left(e_{n}=\left(\delta_{n k}\right)_{k}\right)$ and by $E_{n}$ the span of $\left\{e_{1}, \ldots, e_{n}\right\}$ in $E$. Examples of this are the Minkowski $\ell_{p}$-spaces and $c_{0}$.

Let $f$ be a holomorphic function on some open $0 \in U \subseteq E$ with values in $Y$. The restriction of $f$ to each $U \cap E_{n}$ has a power series expansion $\sum_{\alpha} c_{\alpha}^{(n)}(f) z^{\alpha}$. It is easily seen that $c_{\alpha}^{(n)}(f)=c_{\alpha}^{(n+1)}(f)$ for all $\mathbb{N}_{0}^{n} \subseteq \mathbb{N}_{0}^{n+1}$ and there is a unique family $\left(c_{\alpha}\right)_{\alpha \in \mathbb{N}_{0}^{(\mathbb{N})}}$ of coefficients $\left(\mathbb{N}_{0}^{(\mathbb{N})}\right.$ denotes the set of multi-indices that eventually

Key words and phrases. Monomial series expansion, Holomorphic function, Homogeneous polynomial, Banach space.

Both authors were supported by the MEC Project MTM2008-03211. The second cited author was partially supported by grants PR2007-0384 (MEC) and UPV-PAID-00-07. 
become 0) so that

$$
f(z)=\sum_{\alpha \in \mathbb{N}_{0}^{(\mathbb{N})}} c_{\alpha}(f) z^{\alpha}
$$

In the finite dimensional setting the expression (1) converges for every $z$; in the infinite dimensional setting, however, this is far from being true. Hence, for a given holomorphic function we consider the set on which the monomial expansion converges absolutely (we denote $|z|=\left(\left|z_{n}\right|\right)_{n}$ ):

$$
\operatorname{mon} f=\left\{z \in E: \sum_{\alpha}\left\|c_{\alpha}(f)\right\||z|^{\alpha}<\infty\right\} .
$$

Also, for a given family of holomorphic functions $\mathscr{F}(U, Y)$ we define its set of monomial convergence

$$
\text { mon } \mathscr{F}(U, Y)=\left\{z \in E: \text { for all } f \in \mathscr{F}(U, Y), \sum_{\alpha}\left\|c_{\alpha}(f)\right\||z|^{\alpha}<\infty\right\} .
$$

Sets of monomial convergence of families of scalar valued functions were studied in [8], where the following result [8, Example 4.9] can be found as a particular case of a more general theorem.

\section{Theorem A.}

(1) If $1 \leq r \leq 2$ then $\ell_{1} \cap B_{\ell_{r}} \subseteq \operatorname{mon} H\left(B_{\ell_{r}}\right) \subseteq \ell_{1+\varepsilon} \cap B_{\ell_{r}}$.

(2) If $r \leq 2$ then $\ell_{(1 / r+1 / 2)^{-1}} \cap B_{\ell_{r}} \subseteq \operatorname{mon} H\left(B_{\ell_{r}}\right) \subseteq \ell_{(1 / r+1 / 2)^{-1}+\varepsilon} \cap B_{\ell_{r}}$.

This result includes previous results from $[3,4,2,14,13]$. Our aim is to continue this study, describing the sets of monomial convergence of families of vector valued holomorphic functions in $\ell_{p}$-spaces. In order to do that we have at our disposal the following facts that hold for every Banach sequence space $E$ and every Banach space $Y$ (notation: $E_{0}=\operatorname{span}\left\{e_{k}\right\}_{k=1}^{\infty}$ in $E$, and for $A \subseteq E$ and $B \subseteq F, A \cdot B=$ $\left.\left\{\left(x_{n} y_{n}\right)_{n}:\left(x_{n}\right)_{n} \in A,\left(y_{n}\right)_{n} \in B\right\}\right)$.

$$
\begin{gathered}
\ell_{1} \cap B_{E} \subseteq \operatorname{mon} H_{\infty}\left(B_{E}, Y\right) \\
\left(\operatorname{mon} H_{\infty}\left(B_{\ell_{\infty}}, Y\right) \cdot E_{0}\right) \cap B_{E} \subseteq \text { mon } H_{\infty}\left(B_{E}, Y\right) \\
\ell_{p^{\prime}-\varepsilon} \cap B_{\ell_{\infty}} \subseteq \operatorname{mon} H_{\infty}\left(B_{\ell_{\infty}}, Y\right) \text { whenever } Y \text { has cotype } p .
\end{gathered}
$$

The first one (2) follows from an analysis of the proof of [8, Theorem 4.6] for scalar valued functions, whereas (3) is [6, Lemma 3] and (4) is in [6, page 544] (see below for a definition of cotype). These facts will be some of the key points in proving our main result.

Given $r>1$ we write $r^{\prime}$ for the conjugate of $r$, that is $\frac{1}{r}+\frac{1}{r^{\prime}}=1$. If $r=1$ then we use the convention $\frac{1}{\infty}=0$ and $r^{\prime}=\infty$. 
Theorem 1.1. Let $\mathscr{F}\left(B_{\ell_{r}}, \ell_{q}\right)$ be a set of bounded, holomorphic functions that contains the linear, bounded functions $f: \ell_{r} \rightarrow \ell_{q}$ (restricted to $B_{\ell_{r}}$ ), then

(1) For each $1 \leq r \leq 2$ fixed the following holds.

(a) If $1 \leq q \leq 2$ then

$$
\ell_{1} \cap B_{\ell_{r}} \subseteq \text { mon } \mathscr{F}\left(B_{\ell_{r}}, \ell_{q}\right) \subseteq \ell_{1+\varepsilon} \cap B_{\ell_{r}} \text { for every } \varepsilon>0 .
$$

(b) If $2 \leq q$ then $\ell_{1} \cap B_{\ell_{r}}=$ mon $\mathscr{F}\left(B_{\ell_{r}}, \ell_{q}\right)$.

(2) For each $2 \leq r$ fixed the following holds.

(a) If $1 \leq q \leq 2$ then

$\ell_{(1 / 2+1 / r)^{-1}-\varepsilon} \cap B_{\ell_{r}} \subseteq \operatorname{mon} \mathscr{F}\left(B_{\ell_{r}}, \ell_{q}\right) \subseteq \ell_{(1 / 2+1 / r)^{-1}+\varepsilon} \cap B_{\ell_{r}}$

for every $\varepsilon>0$.

(b) If $2 \leq q \leq r$ then

$\ell_{\left(1 / q^{\prime}+1 / r\right)^{-1}-\varepsilon} \cap B_{\ell_{r}} \subseteq \operatorname{mon} \mathscr{F}\left(B_{\ell_{r}}, \ell_{q}\right) \subseteq \ell_{\left(1 / q^{\prime}+1 / r\right)^{-1}} \cap B_{\ell_{r}}$.

for every $\varepsilon>0$.

(c) If $2 \leq r \leq q$ then $\ell_{1} \cap B_{\ell_{r}}=\operatorname{mon} \mathscr{F}\left(B_{\ell_{r}}, \ell_{q}\right)$.

A mapping $P: X \rightarrow Y$ between Banach spaces is called an $m$-homogeneous polynomial if there exists a continuous $m$-linear $L: X \times \cdots \times X \rightarrow Y$ such that $P(x)=L(x, \ldots, x)$ for every $x \in X$. The space of $m$-homogeneous polynomials between $X$ and $Y$ is denoted by $\mathscr{P}\left({ }^{m} X, Y\right)$; as usual, if $Y=\mathbb{C}$ we simply write $\mathscr{P}\left({ }^{m} X\right)$. The 1-homogeneous polynomials are simply the continuous, linear mappings from $X$ to $Y$; in this case we will write $\mathscr{L}(X, Y)$ for $\mathscr{P}\left({ }^{1} X, Y\right)$. A polynomial (of degree $n$ ) is $P=\sum_{k=0}^{n} P_{k}$, where each $P_{k}$ is a $k$-homogeneous polynomial. A mapping $f: X \rightarrow Y$ is holomorphic if and only if for every $x \in X$ there exists $\rho>0$ and $\left(P_{k}\right)_{k=0}^{\infty}$ (each $P_{k}$ a $k$-homogeneous polynomial) so that $f(x+h)=\sum_{k=0}^{\infty} P_{k}(h)$ for all $\|h\| \leq \rho$.

An $m$-linear mapping $L$ is called symmetric if $L\left(x_{1}, \ldots, x_{m}\right)=L\left(x_{\pi(1)}, \ldots, x_{\pi(m)}\right)$ for every permutation $\pi$ of $\{1, \ldots, m\}$. It is a well known fact [12] that each $m$ homogeneous polynomial has a unique associated symmetric $m$-linear mapping. An $m$-homogeneous polynomial on a Banach sequence space has a monomial expansion and the set of convergence can be considered. In this respect we have the following result [8, Example 4.6]

\section{Theorem B.}

(1) If $2 \leq r$ then $\ell_{\left(\frac{1}{r}+\frac{m-1}{2 m}\right)^{-1}} \subseteq \operatorname{mon} \mathscr{P}\left({ }^{m} \ell_{r}\right) \subseteq \ell_{\left(\frac{1}{r}+\frac{m-1}{2 m}\right)^{-1}+\varepsilon}$. 
(2) If $1<r \leq 2$ then $\ell_{s_{m}} \subseteq \operatorname{mon} \mathscr{P}\left({ }^{m} \ell_{r}\right) \subseteq \ell_{\frac{m r}{r(m-1)+1}+\varepsilon}$ where $s_{m}=\max \left\{1,\left(\frac{1}{r}+\right.\right.$ $\left.\left.\frac{m-1}{2 m}\right)^{-1}\right\}$.

(3) $\ell_{1} \subseteq \operatorname{mon} \mathscr{P}\left({ }^{m} \ell_{1}\right) \subseteq \ell_{1+\varepsilon}$.

We see that in the scalar valued case, the set of monomial convergence of spaces of polynomials depends heavily on the degree. The situation changes strongly in the vector valued setting; [6, Theorem 2] gives that $\ell_{(\cot Y)^{\prime}} \subseteq \operatorname{mon} \mathscr{P}\left({ }^{m} \ell_{\infty}, Y\right) \subseteq$ $\ell_{(\cot Y)^{\prime}+\varepsilon}$ for every $\varepsilon>0$ (see below for a precise definition of $\cot Y$ ). Also, the proof of Theorem 1.1 will show that mon $\mathscr{P}\left({ }^{m} \ell_{r}, \ell_{q}\right)$ does not depend on the degree of the polynomials.

If we consider smaller families of polynomials, namely those taking values in some smaller $\ell_{p}$, then the sets of monomial convergence again depend on the degree of the polynomials. This situation is parallel to that for Dirichlet series already observed in [9]. This parallelism will be studied in detail. We consider $\mathscr{P}_{p}\left({ }^{m} \ell_{r}, \ell_{q}\right)$, the space of $m$-homogeneous polynomials from $\ell_{r}$ to $\ell_{q}$ that take values in some $\ell_{p}$ with $p \leq q$. Then our second main result is

Theorem 1.2. Fix $2 \leq r \leq \infty$. The following hold

(a) If $1 \leq p \leq q \leq 2$ then

$$
\ell_{\left(\frac{m-2(1 / p-1 / q)}{2 m}+\frac{1}{r}\right)^{-1}} \subseteq \operatorname{mon} \mathscr{P}_{p}\left({ }^{m} \ell_{r}, \ell_{q}\right) \subseteq \ell_{\left(\frac{m-2(1 / p-1 / q)}{2 m}+\frac{1}{r}\right)^{-1}+\varepsilon}
$$

for every $\varepsilon>0$.

(b) If $1 \leq p \leq 2 \leq q$ then

$$
\ell_{\left(\frac{m-2(1 / p-1 / 2)}{2 m}+\frac{1}{r}\right)^{-1}} \subseteq \operatorname{mon} \mathscr{P}_{p}\left({ }^{m} \ell_{r}, \ell_{q}\right) \subseteq \ell_{\left(\frac{m-2(1 / p-1 / 2)}{2 m}+\frac{1}{r}\right)^{-1}+\varepsilon}
$$

for every $\varepsilon>0$.

(c) If $2 \leq p \leq r$ then $\ell_{\left(1 / p^{\prime}+1 / r\right)^{-1}}=\operatorname{mon} \mathscr{P}_{p}\left({ }^{m} \ell_{r}, \ell_{q}\right)$.

(d) If $2 \leq r \leq p \leq q$ then $\ell_{1}=$ mon $\mathscr{P}_{p}\left({ }^{m} \ell_{r}, \ell_{q}\right)$.

We recall that a Banach space $X$ is said to have cotype $p$ with $2 \leq p<\infty$ (see [11, Chapter 11]) whenever there is some constant $C>0$ such that for each choice of finitely many vectors $x_{1}, \ldots, x_{n} \in X$ we have

$$
\left(\sum_{i=1}^{n}\left\|x_{i}\right\|^{p}\right)^{1 / p} \leq C\left(\int_{0}^{1}\left\|\sum_{i=1}^{n} \varepsilon_{i}(\omega) x_{i}\right\|^{2} d \omega\right)^{1 / 2},
$$

where $\varepsilon_{i}$ are independent random variables that take values 1 and -1 with probability $1 / 2$; as usual, the smallest such $C$ is denoted by $\mathrm{C}_{p}(X)$. It is well known that $\ell_{p}$ has cotype $\max \{p, 2\}$. We denote by $\cot X$ the infimum over all $p$ 's such that $X$ has cotype $p$. 
Following the notation from $[3,6]$, given a holomorphic function $f$ defined on $U$ and a family $\mathscr{F}$ we define the numbers ( $M$ stands for 'monomial'):

$$
\begin{aligned}
M(f) & =\sup \left\{r: \ell_{r} \cap U \subseteq \operatorname{mon} f\right\} . \\
M(\mathscr{F}(U, Y)) & =\sup \left\{r: \ell_{r} \cap U \subseteq \operatorname{mon} \mathscr{F}(U, Y)\right\} .
\end{aligned}
$$

It is clear from the definition that $M(\mathscr{F}(U, Y))=\inf \{M(f): f \in \mathscr{F}(U, Y)\}$.

With this notation Theorem A implies that $M\left(H_{\infty}\left(B_{\ell_{r}}\right)\right)=1$ if $1 \leq r \leq 2$ and $M\left(H_{\infty}\left(B_{\ell_{r}}\right)\right)=(1 / r+1 / 2)^{-1}$ for $r \geq 2$; on the other hand, from Theorem B, $M\left(\mathscr{P}\left({ }^{m} \ell_{r}\right)\right)=(1 / r+(m-1) /(2 m))^{-1}$ if $r \geq 2$. We see that $M\left(H_{\infty}\left(B_{\ell_{r}}\right)\right)$ and $M\left(\mathscr{P}\left({ }^{m} \ell_{r}\right)\right)$ are different and in the case of the polynomials, $M$ depends on the degree.

In the vector valued setting $\left[6\right.$, Theorem 2] gives that $M\left(H_{\infty}\left(B_{\ell_{\infty}}, Y\right)\right)=M\left(\mathscr{P}\left({ }^{m} \ell_{\infty}, Y\right)\right)=$ $(\cot Y)^{\prime}$ for every infinite dimensional $Y$ and our Theorem 1.1 shows

$$
M\left(H_{\infty}\left(B_{\ell_{r}}, \ell_{q}\right)\right)=M\left(\mathscr{P}\left({ }^{m} \ell_{r}, \ell_{q}\right)\right)= \begin{cases}1 & \text { if } 1 \leq r \leq 2 \\ (1 / 2+1 / r)^{-1} & \text { if } 1 \leq q \leq 2 \leq r \\ (1-1 / q+1 / r)^{-1} & \text { if } 2 \leq q \leq r \\ 1 & \text { if } 2 \leq r \leq q\end{cases}
$$

As we see, the dependence on the degree vanishes in the infinite-dimensional setting. This fact is analyzed in Theorem 1.2. We have from Theorem 1.2 that if $2 \leq r \leq \infty$ then

$$
M\left(\mathscr{P}_{p}\left({ }^{m} \ell_{r}, \ell_{q}\right)\right)= \begin{cases}\left(\frac{m-2(1 / p-1 / q)}{2 m}+\frac{1}{r}\right)^{-1} & \text { if } 1 \leq p \leq q \leq 2 \\ \left(\frac{m-2(1 / p-1 / 2)}{2 m}+\frac{1}{r}\right)^{-1} & \text { if } 1 \leq p \leq 2 \leq q \\ \left(1 / p^{\prime}+1 / r\right)^{-1} & \text { if } 2 \leq p \leq r \\ 1 & \text { if } 2 \leq r \leq p \leq q\end{cases}
$$

The following diagrams show how the different indices $S$ are distributed. Grey parts indicate that the corresponding $S$ is constant and black parts indicate that the corresponding $S$ is not defined. 

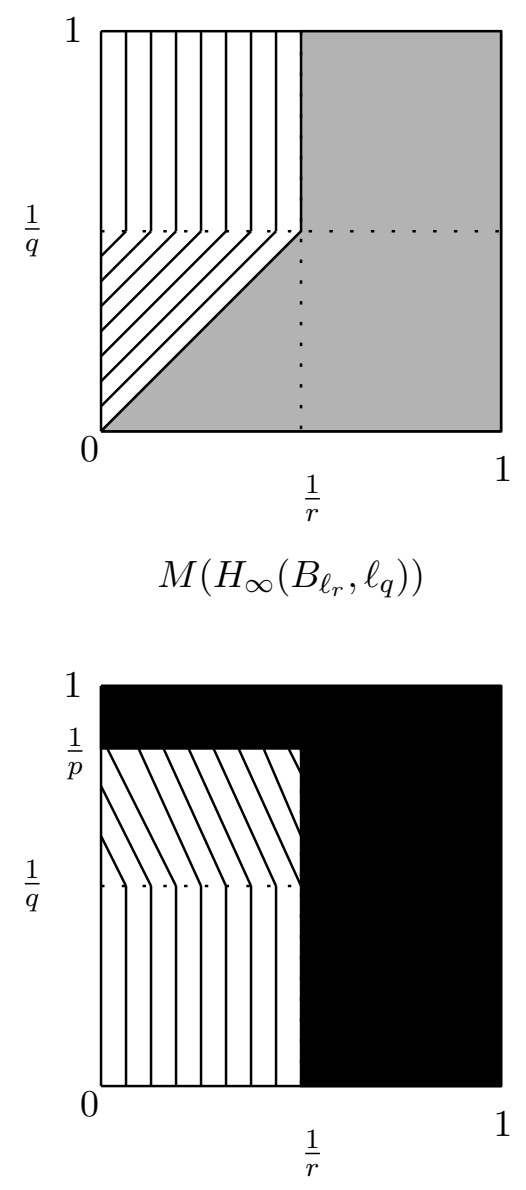

$M\left(\mathscr{P}_{p}\left({ }^{m} \ell_{r}, \ell_{q}\right)\right)$ for $m, p \leq 2$ fixed.

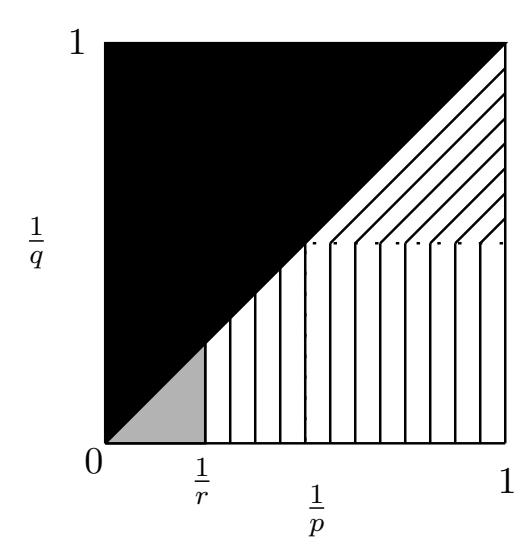

$M\left(\mathscr{P}_{p}\left({ }^{m} \ell_{r}, \ell_{q}\right)\right)$ for $m, r \geq 2$ fixed.

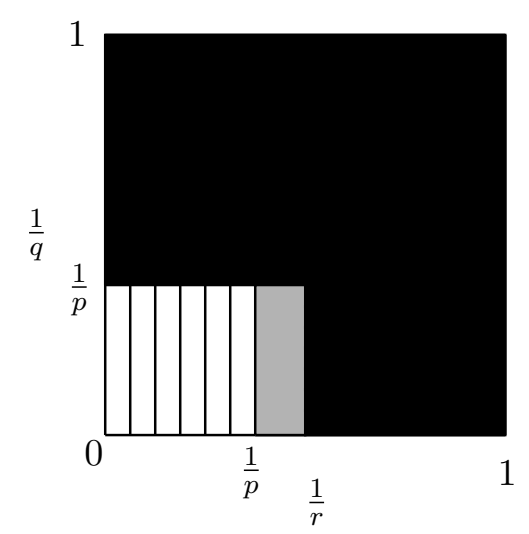

$M\left(\mathscr{P}_{p}\left({ }^{m} \ell_{r}, \ell_{q}\right)\right)$ for $m, p \geq 2$ fixed.

\section{Sets of MONOMial CONVERGENCE OF FAMilies OF HOLOMORPhiC}

FUnCtIONS. The PROOF OF THEOREM 1.1

Let us remark first that if $\mathscr{F}_{2}\left(B_{E}, Y\right) \subseteq \mathscr{F}_{1}\left(B_{E}, Y\right)$ then mon $\mathscr{F}_{1}\left(B_{E}, Y\right) \subseteq$ mon $\mathscr{F}_{2}\left(B_{E}, Y\right)$. We have a set $\mathscr{F}\left(B_{\ell_{r}}, \ell_{q}\right)$ that contains all linear functions and that is contained in the space of bounded, holomorphic functions. Then

$$
\text { mon } H_{\infty}\left(B_{\ell_{r}}, \ell_{q}\right) \subseteq \operatorname{mon} \mathscr{F}\left(B_{\ell_{r}}, \ell_{q}\right) \subseteq \operatorname{mon} \mathscr{L}\left(\ell_{r}, \ell_{q}\right) \cap B_{\ell_{r}}
$$

Therefore, lower inclusions for mon $H_{\infty}\left(B_{\ell_{r}}, \ell_{q}\right)$ will give lower inclusions for mon $\mathscr{F}\left(B_{\ell_{r}}, \ell_{q}\right)$ and upper inclusions for the sets of monomial convergence of the space of linear functions will provide us with the upper inclusions in Theorem 1.1.

We begin with the lower inclusions. Taking $E=\ell_{r}$ and $Y=\ell_{q}$ in (2) we have the lower inclusions in the cases (1a), (1b) and (2c) of Theorem 1.1.

We consider now the case (2a). First of all, if $1 \leq q \leq 2$ and $r=\infty$ we have that $\ell_{q}$ has cotype 2 and then (4) immediately gives the conclusion. On the other hand, if 
$1 \leq q \leq 2 \leq r<\infty$, we fix $\varepsilon>0$ and define $s=(1 / 2+1 / r)^{-1}-\varepsilon$. Then there exists some $u<2$ such that $1 / s=1 / u+1 / r$ and $\ell_{s}=\ell_{u} \cdot \ell_{r}$. If $z \in \ell_{s}$ then there exist $\xi \in \ell_{u}$ and $\zeta \in \ell_{r}$ so that $z=\xi \zeta$. Let $M=1+\sup _{n}\left|\xi_{n}\right|$, hence $\xi / M \in \ell_{u} \cap B_{\ell_{\infty}}$ and $M \zeta \in \ell_{r}$. This implies $\ell_{s} \subseteq\left(\ell_{u} \cap B_{\ell_{\infty}}\right) \cdot \ell_{r}$. Now, since $\ell_{q}$ has cotype 2 , we have from (4) $\ell_{u} \cap B_{\ell_{\infty}} \subseteq$ mon $H_{\infty}\left(B_{\ell_{\infty}}, \ell_{q}\right)$. We apply all this and (3) to finally get

$$
\begin{aligned}
\ell_{s} \cap B_{\ell_{r}} \subseteq\left(\left(\ell_{u} \cap B_{\ell_{\infty}}\right) \cdot \ell_{r}\right) \cap B_{\ell_{r}} \\
\quad \subseteq\left(\left(\operatorname{mon} H_{\infty}\left(B_{\ell_{\infty}}, \ell_{q}\right) \cap B_{\ell_{\infty}}\right) \cdot \ell_{r}\right) \cap B_{\ell_{r}} \subseteq \operatorname{mon} H_{\infty}\left(B_{\ell_{r}}, \ell_{q}\right)
\end{aligned}
$$

The remaining case $(2 \mathrm{~b})$ (i.e $2 \leq q \leq r)$ follows in the same way taking into account that $\ell_{q}$ has cotype $q$.

We look now for the upper inclusions. We consider first the case (2b) (i.e. $2 \leq q \leq r$ ). If $r<\infty$ let $1 / s=1 / q^{\prime}+1 / r$, then $1 / s^{\prime}+1 / r=1 / q$ and $\ell_{s^{\prime}} \cdot \ell_{r}=\ell_{q}$. This shows that, given $\lambda \in \ell_{s^{\prime}}$, the diagonal operator $D_{\lambda}: \ell_{r} \rightarrow \ell_{q}$ defined by $D_{\lambda}(\xi)=\left(\lambda_{n} \xi_{n}\right)_{n}$ is well defined and $D_{\lambda} \in \mathscr{L}\left(\ell_{r}, \ell_{q}\right)$. Clearly $c_{k}\left(D_{\lambda}\right)=\lambda_{k} e_{k}$; hence if $z \in \operatorname{mon} \mathscr{L}\left(\ell_{r}, \ell_{q}\right)$ then

$$
\sum_{k=1}^{\infty}\left|\lambda_{k} z_{k}\right|=\sum_{k=1}^{\infty}\left\|c_{k}\left(D_{\lambda}\right)\right\|\left|z_{k}\right|<\infty .
$$

Since this holds for every $\lambda \in \ell_{s^{\prime}}$ we get that $z \in \ell_{s}$ and mon $\mathscr{L}\left(\ell_{r}, \ell_{q}\right) \subseteq \ell_{\left(1 / q^{\prime}+1 / r\right)^{-1}}$. If $r=\infty$ the result follows in the same way, since the diagonal operator $D_{\lambda}: \ell_{\infty} \rightarrow \ell_{q}$ is always well defined and continuous.

We get (2c) and (1b) from this case. First, if $2 \leq r \leq q$ (this is (2c)) then by means of the inclusion id $: \ell_{r} \hookrightarrow \ell_{q}$ we have that $\mathscr{F}\left(\ell_{r}, \ell_{r}\right) \subseteq \mathscr{F}\left(\ell_{r}, \ell_{q}\right)$. Letting $q=r$ in case $(2 \mathrm{~b})$ we get mon $\mathscr{F}\left(\ell_{r}, \ell_{q}\right) \subseteq$ mon $\mathscr{F}\left(\ell_{r}, \ell_{r}\right)=\ell_{1} \cap B_{\ell_{r}}$.

On the other hand, for (1b) (i.e. $1 \leq r \leq 2$ and $2 \leq q)$, we have $\mathscr{F}\left(\ell_{2}, \ell_{q}\right) \subseteq \mathscr{F}\left(\ell_{r}, \ell_{q}\right)$ (using in this case the inclusion id $: \ell_{r} \hookrightarrow \ell_{2}$ ). Then, by the case $r=2$ in (2b) we have mon $\mathscr{F}\left(\ell_{2}, \ell_{q}\right)=\ell_{1} \cap B_{\ell_{r}}$.

The case (1a) (i.e. $1 \leq r \leq 2$ and $1 \leq q \leq 2$ ) will follow from (2a) since we have $\mathscr{F}\left(\ell_{2}, \ell_{q}\right) \subseteq \mathscr{F}\left(\ell_{r}, \ell_{q}\right)$. Then, as before, mon $\mathscr{F}\left(\ell_{r}, \ell_{q}\right) \subseteq$ mon $\mathscr{F}\left(\ell_{2}, \ell_{q}\right)$ and taking $r=2$ in $(2 \mathrm{a})$ we get mon $\mathscr{F}\left(\ell_{2}, \ell_{q}\right) \subseteq \ell_{1+\varepsilon} \cap B_{\ell_{r}}$ for every $\varepsilon>0$.

We finally consider the case $1 \leq q \leq 2 \leq r$ (this is (2a)). We choose $z \in$ mon $\mathscr{L}\left(\ell_{r}, \ell_{q}\right) \cap B_{\ell_{r}}$ and let us see that for every $\varepsilon>0, z \in \ell_{s+\varepsilon}$ with $1 / s=1 / 2+1 / r$. Since $z \in \operatorname{mon} \mathscr{L}\left(\ell_{r}, \ell_{q}\right)$ we have $\sum_{k}\left\|T e_{k}\right\|_{q}\left|z_{k}\right|<\infty$ for every linear and continuous $T: \ell_{r} \rightarrow \ell_{q}$ or, equivalently, there exists $C>0$ such that

$$
\sum_{k}\left\|T e_{k}\right\|_{q}\left|z_{k}\right| \leq C \sup _{x \in B_{\ell_{r}}}\|T x\|_{q} .
$$


For every $n$ and $k_{1}<\ldots<k_{n}$ we can identify $\left(\operatorname{span}\left\{e_{k_{1}}, \ldots, e_{k_{n}}\right\},\|\|_{u}\right)=\ell_{u}^{n}$, then by Chevét's inequality (see $[15,(43.2)]$ ) there exists a continuous, linear mapping $T_{n}: \ell_{r}^{n} \rightarrow \ell_{q}^{n}$ such that all the $T_{n} e_{k_{j}}$ are elements in $\ell_{q}^{n}$ the entries of which consist only on \pm 1 and such that $\sup _{x \in B_{\ell_{r}^{n}}}\left\|T_{n} x\right\|_{q} \leq K n^{\frac{1}{2}-\frac{1}{r}+\frac{1}{q}}$ for some constant $K>0$ not depending on $n$. Clearly $\left\|T_{n} e_{k_{j}}\right\|_{q}=n^{1 / q}$ for all $k=1, \ldots, n$ and we have from

$$
\frac{1}{n} \sum_{j=1}^{n}\left|z_{k_{j}}\right| \leq C K n^{-(1 / s)} .
$$

Let us see now that $z \in c_{0}$; if this were not the case, there would exist $\delta>0$ and an increasing sequence $\left(k_{j}\right)_{n=1}^{\infty}$ of natural numbers so that $\left|z_{k_{j}}\right|>\delta$ for every $j$. Hence, for each fixed $n$ we have $\sum_{j=1}^{n}\left|z_{k_{j}}\right|>n \delta$. But this contradicts the fact that the right-hand side of (9) tends to 0 . Thus there is some bijection $\sigma: \mathbb{N} \rightarrow \mathbb{N}$ such that $z_{n}^{\downarrow}=z_{\sigma(n)}$. Let us take $k_{1}<\cdots<k_{n}$ so that $\{\sigma(1), \ldots, \sigma(n)\}=\left\{k_{1}, \ldots, k_{n}\right\}$; then

$$
0 \leq z_{n}^{\downarrow} \leq \frac{1}{n} \sum_{j=1}^{n}\left|z_{\sigma(j)}\right|=\frac{1}{n} \sum_{j=1}^{n}\left|z_{k_{j}}\right| \leq \frac{C K}{n^{1 / s}} .
$$

This implies $\sum_{n}\left|z_{n}\right|^{s+\varepsilon} \leq C K \sum_{n} n^{-(1+\varepsilon / s)}<\infty$ and $z \in \ell_{s+\varepsilon}$ for every $\varepsilon>0$. This completes the case (2a) and the proof of Theorem 1.1.

Note that letting $r=\infty$ we again get the results for $H_{\infty}\left(B_{\ell_{\infty}}, \ell_{q}\right)$ we already know from [6].

\section{Sets of monomial convergence of spaces of homogeneous Polynomials. The PRoOF of TheOREM 1.2}

Given a Banach sequence space $E$, Banach spaces $X$ and $Y$ and an operator $v: X \rightarrow Y$ we consider the space of all $m$-homogeneous polynomials from $E$ to $Y$ that factor through $v$, that is

$$
\mathscr{P}_{v}\left({ }^{m} E, Y\right)=\left\{P \in \mathscr{P}\left({ }^{m} E, Y\right): \text { there exists } Q \in \mathscr{P}\left({ }^{m} E, X\right), P=v Q\right\} .
$$

Each polynomial $P$ has a monomial expansion $\sum_{|\alpha|=m} c_{\alpha}(P) z^{\alpha}$ and we consider mon $\mathscr{P}_{v}\left({ }^{m} E, Y\right)$, the set of monomial convergence. Let us note that by a simple closed graph argument the space $\mathscr{P}_{p}\left({ }^{m} \ell_{r}, \ell_{q}\right)$ is simply $\mathscr{P}_{v}\left({ }^{m} \ell_{r}, \ell_{q}\right)$ from (10) when we consider $v=\operatorname{id}_{p, q}: \ell_{p} \hookrightarrow \ell_{q}$. We again begin with the lower inclusions. In the same spirit as in [8, Theorem 3.7] and [6, Lemma 3] we have that for every Banach sequence space $E$ and every operator $v$ from $X$ to $Y$ the following holds

$$
\operatorname{mon} \mathscr{P}_{v}\left({ }^{m} \ell_{\infty}, Y\right) \cdot E \subseteq \operatorname{mon} \mathscr{P}_{v}\left({ }^{m} E, Y\right) \text {. }
$$

Indeed, let $z=w_{0} \cdot u \in \operatorname{mon} \mathscr{P}_{v}\left({ }^{m} \ell_{\infty}, Y\right) \cdot E$. Given any $P \in \mathscr{P}_{v}\left({ }^{m} E, Y\right)$, there exists $Q \in \mathscr{P}\left({ }^{m} E, X\right)$ such that $P=v Q$ and we define $Q_{u}: \ell_{\infty} \rightarrow X$ by $Q_{u}(w)=$ 
$Q(u \cdot w)=\sum_{|\alpha|=m} c_{\alpha}(Q) u^{\alpha} w^{\alpha}$. Clearly this is well defined and is an $m$-homogeneous polynomial on $\ell_{\infty}$ such that $c_{\alpha}\left(Q_{u}\right)=c_{\alpha}(Q) u^{\alpha}$. Then

$$
\sum_{|\alpha|=m}\left\|c_{\alpha}(P)\right\||z|^{\alpha}=\sum_{|\alpha|=m}\left\|c_{\alpha}(v Q)\right\||u|^{\alpha}\left|w_{0}\right|^{\alpha}=\sum_{|\alpha|=m}\left\|v\left(c_{\alpha}(Q)\right) u^{\alpha}\right\|\left|w_{0}\right|^{\alpha} .
$$

This is finite since $w_{0} \in \operatorname{mon} \mathscr{P}_{v}\left({ }^{m} \ell_{\infty}, Y\right)$ and gives (11). Hence, taking $Y=\ell_{q}$, $E=\ell_{r}$ and $v=\mathrm{id}: \ell_{p} \hookrightarrow \ell_{q}$ we have

$$
\operatorname{mon} \mathscr{P}_{p}\left({ }^{m} \ell_{\infty}, \ell_{q}\right) \cdot \ell_{r} \subseteq \operatorname{mon} \mathscr{P}_{p}\left({ }^{m} \ell_{r}, \ell_{q}\right) \text {. }
$$

We need then lower estimates for mon $\mathscr{P}_{p}\left({ }^{m} \ell_{\infty}, \ell_{q}\right)$. Let us note that if $\ell_{\mu} \subseteq$ mon $\mathscr{P}_{p}\left({ }^{m} \ell_{\infty}, \ell_{q}\right)$ then we have

$$
\ell_{s}=\ell_{\mu} \cdot \ell_{r} \subseteq \operatorname{mon} \mathscr{P}_{p}\left({ }^{m} \ell_{r}, \ell_{q}\right) \text { with } \frac{1}{s}=\frac{1}{\mu}+\frac{1}{r} .
$$

Following [10] we say that $v: X \rightarrow Y$ is a $(r, 1)$-summing operator of order $m$ if there exists a constant $C>0$ such that

$$
\left(\sum_{|\alpha|=m}\left\|v c_{\alpha}(Q)\right\|^{r}\right)^{1 / r} \leq C\|Q\|
$$

for every $Q \in \mathscr{P}\left({ }^{m} \ell_{\infty}, X\right)$. This concept is closely related to sets of monomial convergence, as the following result shows

Proposition 3.1. If $v: X \rightarrow Y$ is $(r, 1)$-summing of order $m$ then $\ell_{r^{\prime}} \subseteq$ mon $\mathscr{P}_{v}\left({ }^{m} \ell_{\infty}, Y\right)$.

Proof. Let $C>0$ be such that for every $Q \in \mathscr{P}\left({ }^{m} \ell_{\infty}, X\right)$ we have $\left(\sum_{\alpha}\left\|c_{\alpha}(v Q)\right\|^{r}\right)^{1 / r} \leq$ $C\|Q\|$. Now, if $z \in B_{\ell_{r^{\prime}}}$ we can apply Hölder's inequality to get

$$
\begin{aligned}
\sum_{|\alpha|=m}\left\|c_{\alpha}(v Q)\right\|\left|z^{\alpha}\right| & \leq\left(\sum_{|\alpha|=m}\left\|c_{\alpha}(v Q)\right\|^{r}\right)^{1 / r}\left(\sum_{|\alpha|=m}\left|z^{\alpha}\right|^{r^{\prime}}\right)^{1 / r^{\prime}} \\
& \leq C\|Q\|\left(\sum_{|\alpha|=m}\left|z^{r^{\prime}}\right|^{\alpha}\right)^{1 / r^{\prime}}
\end{aligned}
$$

Let us recall now that $\sum_{\alpha \in \mathbb{N}^{(\mathbb{N})}}\left|\omega^{\alpha}\right|<\infty$ if and only if $\omega \in \ell_{1} \cap \mathbb{D}^{\mathbb{N}}$. This implies that the right-hand side of (13) is finite since $z \in B_{\ell_{r^{\prime}}}$. Hence $B_{\ell_{r^{\prime}}} \subseteq$ mon $\mathscr{P}_{v}\left({ }^{m} \ell_{\infty}, Y\right)$ and then $\ell_{r^{\prime}} \subseteq \operatorname{mon} \mathscr{P}_{v}\left({ }^{m} \ell_{\infty}, Y\right)$.

From [10, Lemma 3] we know that every $(p, 1)$-summing operator $(1 \leq p \leq 2)$ taking values on a cotype 2 space is $(\rho, 1)$-summing of order $m$ with $\rho=\frac{2 m}{m+2(1 / p-1 / 2)}$. As a consequence of this and Proposition 3.1 we have that if $v$ is $(p, 1)$-summing with $1 \leq p \leq 2$ and takes values in a cotype 2 space then

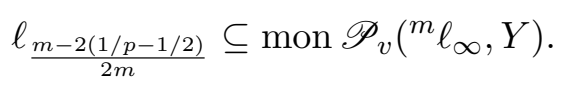

When $1 \leq p \leq q \leq 2$ (this is case (a)) we know from the Bennett-Carl inequalities (see $[1,5])$ that $\operatorname{id}_{p, q}$ is $(\rho, 1)$-summing, where $\frac{1}{\rho}=\frac{1}{p}-\frac{1}{q}+\frac{1}{2}$. Then (12) and (14) give $\frac{1}{s}=\frac{m-2(1 / p-1 / q)}{2 m}+\frac{1}{r}$; note that, since $r \geq 2$, we have $\frac{1}{s} \leq \frac{1}{2}-\frac{1}{m}\left(\frac{1}{p}-\frac{1}{q}\right)+\frac{1}{2}<1$ 
and $s>1$.

The lower inclusion in case (b) follows from (12) and Proposition 3.1 and the fact that $\operatorname{id}_{p, q}$ is $\left(\frac{2 m}{m+2(1 / p-1 / 2)}, 1\right)$-summing of order $m$ if $1 \leq p \leq 2 \leq q$ [10, Theorem 1]. For the case (c) (i.e. $2 \leq p \leq r)\left[10\right.$, Theorem 1] gives that $\operatorname{id}_{p, q}$ is $(p, 1)$-summing of order $m$ and the lower inclusion follows again from (12) and Proposition 3.1. In both cases we have that the corresponding spaces are strictly bigger than $\ell_{1}$ (except when $2 \leq p=r$; in this case, it equals $\ell_{1}$ ).

The lower bound for case (d) follows from (2).

Getting the upper inclusion in case (a) will require some work; we will treat this later. The upper inclusion for (b) (i.e. $1 \leq p \leq 2 \leq q$ ) will follow from (a), since $2 \leq q$ implies that $\mathscr{P}_{p}\left({ }^{m} \ell_{r}, \ell_{2}\right) \subseteq \mathscr{P}_{p}\left({ }^{m} \ell_{r}, \ell_{q}\right)$ and this yields mon $\mathscr{P}_{p}\left({ }^{m} \ell_{r}, \ell_{q}\right) \subseteq$ mon $\mathscr{P}_{p}\left({ }^{m} \ell_{r}, \ell_{2}\right)$.

In order to give an upper inclusion for (c) (this is the case $2 \leq p \leq r$ ), let us define for each $\lambda \in \ell_{s^{\prime}}$ the diagonal operator $D_{\lambda}: \ell_{r} \rightarrow \ell_{p}$ by $\xi \rightsquigarrow\left(\lambda_{n} \xi_{n}\right)_{n}$. Since $1 / p=$ $1 / s^{\prime}+1 / r$ we have $\ell_{p}=\ell_{s^{\prime}} \ell_{r}$ and $D_{\lambda} \in \mathscr{L}\left(\ell_{r}, \ell_{p}\right)$. Now, if $z \in \operatorname{mon} \mathscr{L}_{p}\left(\ell_{r}, \ell_{p}\right)$ then $\sum_{n}\left\|c_{n}\left(D_{\lambda}\right)\right\|_{q}\left|z_{n}\right|<\infty$. The fact that this holds for every $\lambda$ and that $c_{n}\left(D_{\lambda}\right)=\lambda_{n}$ give that $z \in \ell_{s}$. Hence mon $\mathscr{P}_{p}\left({ }^{m} \ell_{r}, \ell_{q}\right) \subseteq \operatorname{mon} \mathscr{L}_{p}\left(\ell_{r}, \ell_{p}\right) \subseteq \ell_{s}$.

In the remaining case (d) (that is $2 \leq r \leq p \leq q$ ) we clearly have mon $\mathscr{P}_{p}\left({ }^{m} \ell_{r}, \ell_{q}\right) \subseteq$ mon $\mathscr{P}\left({ }^{m} \ell_{r}, \ell_{p}\right)$ and the result follows from Theorem 1.1.

We focus now in case (a) (i.e. $1 \leq p \leq q \leq 2$ ). The general idea is the same as that we used for the upper inclusion in case (2a) in Theorem 1.1: to show that the set of monomial convergence is contained in certain Lorentz space by means of getting proper upper bounds for $\frac{1}{n} \sum_{j}\left|z_{k_{j}}\right|$. However, the tools and techniques are in this case more sophisticated. We follow some of the ideas in [8], and adopt a general point of view. The next result is modelled along [8, Lemma 4.1], [6, Lemma 1].

Lemma 3.2. Let $E$ be a Banach sequence space and $v \neq 0$ an operator between Banach spaces $X$ and $Y$. Then for every $z \in$ mon $\mathscr{P}_{v}\left({ }^{m} E, Y\right)$ there exists a constant $K_{v, z}>0$ such that for every $P \in \mathscr{P}_{v}\left({ }^{m} E, Y\right)$,

$$
\sum_{|\alpha|=m}\left\|c_{\alpha}(P)\right\|\left|z^{\alpha}\right| \leq K_{v, z} \inf \left\{\|Q\|: Q \in \mathscr{P}\left({ }^{m} E, X\right), P=v Q\right\} .
$$

Proof. Let $z \in \operatorname{mon} \mathscr{P}_{v}\left({ }^{m} E, Y\right)$ and for each $n$ we define

$$
\mathfrak{F}_{n}=\left\{Q \in \mathscr{P}\left({ }^{m} E, X\right): \sum_{|\alpha|=m}\left\|c_{\alpha}(v Q)\right\|\left|z^{\alpha}\right| \leq n\right\}
$$


Since both $v$ and $c_{\alpha}$ (seen as a linear mapping on $\mathscr{P}\left({ }^{m} E, Y\right)$ with values in $Y$ ) are continuous, each $\mathfrak{F}_{n}$ is closed in $\mathscr{P}\left({ }^{m} E, X\right)$. On the other hand, the fact that $z \in$ mon $\mathscr{P}_{v}\left({ }^{m} E, Y\right)$ gives that $\bigcup_{n} \mathfrak{F}_{n}=\mathscr{P}\left({ }^{m} E, X\right)$. Then, by Baire's Theorem there exist $N \in \mathbb{N}, Q_{0} \in \mathscr{P}\left({ }^{m} E, X\right)$ and $s>0$ so that

$$
Q_{0}+s B_{\mathscr{P}\left({ }^{m} E, X\right)} \subseteq \mathfrak{F}_{N} .
$$

Now, given $P=v Q \in \mathscr{P}_{v}\left({ }^{m} E, Y\right)$ we have $c_{\alpha}(Q)=\frac{\|Q\|}{s}\left(c_{\alpha}\left(Q_{0}+\frac{s Q}{\|Q\|}\right)-c_{\alpha}\left(Q_{0}\right)\right)$, hence

$$
\begin{aligned}
& \sum_{|\alpha|=m}\left\|c_{\alpha}(P)\right\|\left|z^{\alpha}\right|=\sum_{|\alpha|=m}\left\|c_{\alpha}(v Q)\right\|\left|z^{\alpha}\right| \\
& \quad \leq \frac{\|Q\|}{s}\left(\sum_{|\alpha|=m}\left\|c_{\alpha}\left(v\left(Q_{0}+\frac{s Q}{\|Q\|}\right)\right)\right\|\left|z^{\alpha}\right|+\sum_{|\alpha|=m}\left\|c_{\alpha}\left(v Q_{0}\right)\right\|\left|z^{\alpha}\right|\right) \leq \frac{2 N}{s}\|Q\| .
\end{aligned}
$$

This completes the proof of the Lemma.

Following $[7,8]$ we consider now the arithmetic Bohr radius

$$
\begin{gathered}
A\left(\mathscr{P}_{v}\left({ }^{m} E_{n}, Y\right), \lambda\right)=\sup \left\{\frac{1}{n} \sum_{i=1}^{n} t_{i}: t \in \mathbb{R}_{\geq 0}^{n} \text { such that for all } Q \in \mathscr{P}\left({ }^{m} E_{n}, X\right),\right. \\
\left.\qquad \sum_{|\alpha|=m}\left\|c_{\alpha}(v Q)\right\| t^{\alpha} \leq \lambda\|Q\|\right\} .
\end{gathered}
$$

As it happens in [8], getting upper bounds for the arithmetic Bohr radius will help us to get upper inclusions for sets of monomial convergence. We will do this in two steps. As a first step we have that for every $z \in \operatorname{mon} \mathscr{P}_{v}\left({ }^{m} E, Y\right)$ there exists $\lambda \geq 1$ such that for every $n$ and every choice of natural numbers $k_{1}<\cdots<k_{n}$

$$
\frac{1}{n} \sum_{j=1}^{n}\left|z_{k_{j}}\right| \leq A\left(\mathscr{P}_{v}\left({ }^{m} E_{n}, Y\right), \lambda\right) .
$$

This is proved following exactly the same steps as [8, Lemma 4.2] using Lemma 3.2. For our second step we fix $1 \leq q \leq 2 \leq r, m \in \mathbb{N}$ and $\lambda \geq 1$ and we take as operator $v$ the inclusion $\operatorname{id}_{p, q}: \ell_{p} \hookrightarrow \ell_{q}$ for some $p \leq q$. We then have that there exists some constant $C>0$ (independent from $\lambda, p, q$ and $r$ ) such that

$$
A\left(\mathscr{P}_{\operatorname{id}_{p, q}}\left({ }^{m} \ell_{r}^{n}, \ell_{q}\right), \lambda\right) \leq C \lambda^{1 / m} n^{-\left(\frac{m-2(1 / p-1 / q)}{2 m}+\frac{1}{r}\right)} .
$$

In order to prove this inequality we choose $t \in \mathbb{R}_{\geq 0}^{n}$ such that for every $P \in$ $\mathscr{P}_{\operatorname{id}_{p, q}}\left({ }^{m} \ell_{r}, \ell_{q}\right)$

$$
\sum_{|\alpha|=m}\left\|c_{\alpha}(P)\right\|_{q} t^{\alpha} \leq \lambda\|P\|_{\mathscr{P}\left(m \ell_{r}, \ell_{p}\right)} .
$$

We take now independent gaussian random variables $g_{j}$ with $j=1, \ldots, n$. It is a well known fact (see e.g. [15]) that there exists a universal constant $M>0$ such 
that $\int\left\|\sum_{j=1}^{n} g_{j}(\omega) e_{j}\right\|_{u} d \omega \leq M n^{1 / u}$. We know from [9, Lemma 4.2] that there exists a constant $K_{m}>0$ depending only on $m$ such that for every choice of scalars $\left(\lambda_{\alpha}\right)_{\alpha}$ there exist $c_{\alpha} \in \ell_{p}^{n}$ whose entries consist only of \pm 1 satisfying

$$
\begin{aligned}
& \left\|\sum_{|\alpha|=m} \lambda_{\alpha} c_{\alpha} z^{\alpha}\right\|_{\mathscr{P}\left(m \ell_{r}^{n}, \ell_{p}^{n}\right)} \leq K_{m}\left(\sup _{|\alpha|=m}\left|\lambda_{\alpha}\right| \sqrt{\frac{\alpha !}{m !}}\right) \\
& \left(\left\|\mathrm{id}: \ell_{r}^{n} \rightarrow \ell_{2}^{n}\right\|^{m-1} \| \text { id }: \ell_{2}^{n} \rightarrow \ell_{p}^{n}\left\|\int\right\| \sum_{j=1}^{n} g_{j}(\omega) e_{j} \|_{r^{\prime}} d \omega\right. \\
& \left.\quad+\| \text { id }: \ell_{r}^{n} \rightarrow \ell_{2}^{n}\left\|^{m} \int\right\| \sum_{j=1}^{n} g_{j}(\omega) e_{j} \|_{p} d \omega\right) \\
& \leq K_{m, 2}\left(\sup _{|\alpha|=m}\left|\lambda_{\alpha}\right| \sqrt{\frac{\alpha !}{m !}}\right)\left(\left(n^{1 / 2-1 / r}\right)^{m-1} n^{1 / p-1 / 2} n^{1 / r^{\prime}}+\left(n^{1 / 2-1 / r}\right)^{m} n^{1 / p}\right) \\
& =2 K_{m, 2}\left(\sup _{|\alpha|=m}\left|\lambda_{\alpha}\right| \sqrt{\frac{\alpha !}{m !}}\right)\left(n^{1 / 2-1 / r}\right)^{m} n^{1 / p} .
\end{aligned}
$$

Taking now $\lambda_{\alpha}=m ! / \alpha$ ! and choosing the corresponding $c_{\alpha} \in \ell_{p}^{n}$ we get

$$
\begin{aligned}
& \left(\sum_{j=1}^{n} t_{j}\right)^{m}=\sum_{|\alpha|=m} \frac{m !}{\alpha !} t^{\alpha}=\frac{1}{n^{1 / q}} \sum_{|\alpha|=m}\left\|c_{\alpha}\right\|_{\ell_{q}} \frac{m !}{\alpha !} t^{\alpha} \\
& \leq \frac{\lambda}{n^{1 / q}}\left\|\sum_{|\alpha|=m} c_{\alpha} \frac{m !}{\alpha !} z^{\alpha}\right\|_{\mathscr{P}\left(m \ell_{r}^{n}, \ell_{p}^{n}\right)} \leq 2 K_{m, 2} \lambda \sup _{|\alpha|=m} \sqrt{\frac{m !}{\alpha !}} \frac{1}{n^{1 / q}}\left(n^{1 / 2-1 / r}\right)^{m} n^{1 / p} .
\end{aligned}
$$

Taking the $m$-th root and dividing by $n$ gives (17) with $C=\left(2 K_{m, 2} \sup _{|\alpha|=m} \sqrt{\frac{m !}{\alpha !}}\right)^{\frac{1}{m}}$.

We are now ready to prove the upper inclusion we aimed at. Let $z \in \operatorname{mon} \mathscr{P}_{p}\left({ }^{m} \ell_{r}, \ell_{q}\right)$. Using (16) and (17) we can find some $\lambda \geq 1$ such that for any choice of natural numbers $k_{1}<\cdots<k_{n}$

$$
\frac{1}{n} \sum_{j=1}^{n}\left|z_{k_{j}}\right| \leq A\left(\mathscr{P}\left({ }^{m} \ell_{r}^{n}, \ell_{q}\right), \lambda\right) \leq C \lambda^{1 / m} n^{-\left(\frac{m-2(1 / p-1 / q)}{2 m}+\frac{1}{r}\right)} .
$$

Proceeding as in the case (2a) of Theorem 1.1 it is proved that the decreasing rearrangement $z^{\downarrow}$ satisfies $\sup _{n} z_{n}^{\downarrow} n^{\frac{m-2(1 / p-1 / q)}{2 m}+\frac{1}{r}} \leq K$. Therefore $z$ is in the Lorentz space $\ell_{\left(\frac{m-2(1 / p-1 / q)}{2 m}\right)^{-1}, \infty} \subseteq \ell_{\left(\frac{m-2(1 / p-1 / q)}{2 m}\right)^{-1}+\varepsilon}$ for every $\varepsilon>0$. This completes the proof of (a) and hence of Theorem 1.2.

Remark 3.3. Let us note that letting $p=q$ in the previous result improves slightly some of the lower inclusions for spaces of $m$-homogeneous polynomials given in Theorem 1.1. More precisely we have for $2 \leq r$ that $\ell_{(1 / 2+1 / r)^{-1}} \subseteq$ mon $\mathscr{P}\left({ }^{m} \ell_{r}, \ell_{q}\right) \subseteq$ $\ell_{(1 / 2+1 / r)^{-1}+\varepsilon}$ for every $\varepsilon>0$ if $1 \leq q \leq 2$, and $\ell_{\left(1 / q^{\prime}+1 / r\right)^{-1}}=$ mon $\mathscr{P}\left({ }^{m} \ell_{r}, \ell_{q}\right)$ when $2 \leq q \leq r$. 
Remark 3.4. Given $v: X \rightarrow Y$, a non-zero operator between Banach spaces, $B H_{m}(v)$ is defined in [10] as the infimum over all $r$ so that $v$ is $(r, 1)$-summing of order $m$. Then Proposition 3.1 gives that $M\left(\mathscr{P}_{v}\left({ }^{m} \ell_{\infty}, Y\right)\right) \geq B H_{m}(v)^{\prime}$ for every $m$. A natural question now is: Is it true that $M\left(\mathscr{P}_{v}\left({ }^{m} \ell_{\infty}, Y\right)\right)=B H_{m}(v)^{\prime}$ for every $m$ ? Comparing Proposition 1.2 with [10, Theorem 1] we have a positive answer to this problem when $v=\mathrm{id}_{p, q}$. We can also give positive answers in two more situations.

First of all, in the general case for $m=1: S_{1}\left(\mathscr{P}_{v}\left({ }^{m} \ell_{\infty}, Y\right)\right)=B H_{1}(v)^{\prime}$ holds for every operator $v \neq 0$. Indeed, let $r$ be such that $\ell_{r} \subseteq \operatorname{mon} \mathscr{L}_{v}\left(\ell_{\infty}, Y\right)$. For any operator $A: \ell_{\infty} \rightarrow X$ with coefficients $c_{k}$ we have that $\sum_{k}\left\|v c_{k} z_{k}\right\|<\infty$ for every $z \in \ell_{r}$. This implies that $\left(\left\|v c_{k}\right\|\right)_{k} \in \ell_{r^{\prime}}$. Hence the mapping $\mathscr{L}\left(\ell_{\infty}, X\right) \rightarrow \ell_{r^{\prime}}(Y)$ that maps $A$ to $\left(v c_{k}\right)$ is well defined. A simple closed-graph argument shows that it is continuous; hence there is a constant $c>0$ such that $\sum_{k}\left\|v c_{k}\right\| \leq c\|A\|$ for every $A$. This gives that $r^{\prime} \geq B H_{1}(v)$ and the conclusion.

We have another positive answer when $v$ is $(p, 1)$-summing for every $p>1$ (or 1 summing). Indeed, on one hand we have that for every non zero operator $v: X \rightarrow Y$ and every $m$ the following holds

$$
\text { mon } \mathscr{P}_{v}\left({ }^{m} E, Y\right) \subseteq \ell_{\frac{2 m}{m-1}+\varepsilon} \text { for all } \varepsilon>0 \text {. }
$$

Indeed, given $P \in \mathscr{P}\left({ }^{m} E\right)$ we can define $\hat{P} \in \mathscr{P}_{v}\left({ }^{m} E ; Y\right)$ by fixing $x_{0} \in X$ such that $v\left(x_{0}\right) \neq 0$ and doing $\hat{P}(x)=P(x) v\left(x_{0}\right)$. By means of this identification we can consider $\mathscr{P}\left({ }^{m} E\right) \subseteq \mathscr{P}_{v}\left({ }^{m} E ; Y\right)$. Hence, by [8, Theorem 4.5(i)]

$$
\text { mon } \mathscr{P}_{v}\left({ }^{m} E, Y\right) \subseteq \text { mon } \mathscr{P}\left({ }^{m} E\right) \subseteq \ell_{\frac{2 m}{m-1}+\varepsilon} \text { for all } \varepsilon>0 .
$$

On the other hand, if $v$ is $(p, 1)$-summing for every $p>1$ and takes values in a cotype 2 space (or if it is 1 -summing) then by [10, Lemma 3] it is $\left(\frac{2 m}{m+1}, 1\right)$-summing of order $m$. Then by Proposition 3.1 we have

$$
\begin{gathered}
\ell_{\frac{2 m}{m-1}} \subseteq \text { mon } \mathscr{P}_{v}\left({ }^{m} \ell_{\infty}, Y\right) \subseteq \ell_{\frac{2 m}{m-1}+\varepsilon} \text { for all } \varepsilon>0 \text { and } \\
M\left(\mathscr{P}_{v}\left({ }^{m} \ell_{\infty}, Y\right)\right)=\frac{2 m}{m-1}=B H_{m}(v)^{\prime} .
\end{gathered}
$$

\section{Monomial Convergence And Dirichlet Series}

As we have already mentioned, the study of sets of convergence of monomial expansions in infinitely many variables was closely related to the problem of the convergence of Dirichlet series. It all goes back to H.Bohr who, in [3, 4] considered for a Dirichlet series $\sum_{n} a_{n} / n^{s}$ the abscissas of absolute convergence $\left(\sigma_{a}\right)$ and of uniform convergence $\left(\sigma_{u}\right)$ and defined the number $S=\sup \sigma_{a}-\sigma_{u}$, where the supremum ranges over all Dirichlet series. This number $S$ gives the maximal width of the strip ( $S$ stands then for 'strip') on which a Dirichlet series can converge 
uniformly but not absolutely. Bohr proved that $S=1 / M\left(H_{\infty}\left(B_{\ell_{\infty}}\right)\right)$ and by giving a lower bound to $M\left(H_{\infty}\left(B_{\ell_{\infty}}\right)\right)$ he showed that $S \leq 1 / 2$. The job was finished by Bohnenblust and Hille who in [2] computed the precise value of $M\left(\mathscr{P}\left({ }^{m} \ell_{\infty}\right)\right)$; this gave lower bounds for $M\left(H_{\infty}\left(B_{\ell_{\infty}}\right)\right)$ and finally $S=1 / 2$.

The vector valued case was studied in [6]. There Dirichlet series $\sum_{n} a_{n} / n^{s}$ are considered with $a_{n} \in X$, where $X$ is some Banach space. Again, each Dirichlet series has abscissas of uniform and absolute convergence; then $S(X)=\sup \sigma_{a}-\sigma_{u}$ (the supremmum ranging over all the Dirichlet series on $X$ ) gives the width of the maximal strip on which a series can converge uniformly but not absolutely. Also in this case we have [6, Theorem 3] that $S(X)=1 / M\left(H_{\infty}\left(B_{\ell_{\infty}}, X\right)\right)$. The precise value of $M\left(H_{\infty}\left(B_{\ell_{\infty}}, X\right)\right)$ is computed in [6, Theorem 2] and then the precise value of $S(X)=1-\frac{1}{\cot X}$ is given [6, Theorem 1 ].

We see that in both cases the theory of Dirichlet series and of sets of monomial convergence of holomorphic functions on $B_{\ell_{\infty}}$ are very closely related. For any nonzero operator $v: X \rightarrow Y$ between Banach spaces the number $S_{m}(v)=\sup \sigma_{a}^{Y}-\sigma_{u}^{X}$ is defined in [9]. Now, the supremmum is considered over all the $m$-homogeneous Dirichlet polynomials on $X$ (i.e. Dirichlet series $\sum a_{n} / n^{s}$ for which $a_{n}$ is different from 0 only if $n$ has a prime number decomposition with exactly $m$ factors), $\sigma_{u}^{X}$ is the abscissa of uniform convergence of such a Dirichlet polynomial in $X$ and $\sigma_{a}^{Y}$ is the abscissa of absolute convergence of $\sum v\left(a_{n}\right) / n^{s}$ in $Y$. Our aim now is to show that also in this case there is the same kind of relationship between Dirichlet series and sets of monomial convergence expressed in the following result.

Theorem 4.1. For any operator $v \neq 0$ we have

$$
S_{m}(v)=\frac{1}{\left.M\left(\mathscr{P}_{v}{ }^{(m} \ell_{\infty}, Y\right)\right)} .
$$

We follow the same trends as in [6]. We begin with an analogue to [6, Lemma 2]; due to our particular setting, the proof can be simplified.

Lemma 4.2. Let $E$ be a Banach sequence space and $v \neq 0$ an operator between Banach spaces $X$ and $Y$. Let $\omega=\left(\omega_{n}\right)_{n} \in \operatorname{mon} \mathscr{P}_{v}\left({ }^{m} E, Y\right)$ and $z=\left(z_{n}\right)_{n} \in E$ so that $\left|z_{n}\right| \leq\left|\omega_{n}\right|$ for all but finitely many $n$. Then $z \in \operatorname{mon} \mathscr{P}_{v}\left({ }^{m} E, Y\right)$.

Proof. Let us choose $r$ so that $\left|z_{n}\right| \leq\left|\omega_{n}\right|$ for all $n \geq r$. We fix $P \in \mathscr{P}_{v}\left({ }^{m} E, Y\right)$ and $Q \in \mathscr{P}\left({ }^{m} E, X\right)$ such that $P=v Q$. Let $T: E \times \cdots \times E \rightarrow X$ be the symmetric $m$-linear mapping associated to $Q$. For each choice $n_{1}, \ldots, n_{r} \in \mathbb{N}_{0}$ such that $n_{1}+$ $\cdots+n_{r}=N \leq m$ and $x_{1}, \ldots, x_{m-N} \in E$,

$$
T_{n_{1}, \ldots, n_{r}}\left(x_{1}, \ldots, x_{m-N}\right)=T\left(e_{1}, ._{1}^{n_{1}}, e_{1}, \ldots, e_{n},{ }^{n_{n}} ., e_{n}, x_{1}, \ldots, x_{m-N}\right) .
$$


Clearly $T_{n_{1}, \ldots, n_{r}}$ is a symmetric $(m-N)$-linear mapping from $E$ to $X$; let $Q_{n_{1}, \ldots, n_{r}}$ be the associated polynomial. We have

$$
c_{\alpha}\left(Q_{n_{1}, \ldots, n_{r}}\right)= \begin{cases}c_{\left(n_{1}, \ldots, n_{r}, \alpha_{r+1}, \alpha_{r+2}, \ldots\right)}(Q) & \text { if } \alpha=\left(0, \ldots, 0, \alpha_{r+1}, \alpha_{r+2}, \ldots\right) \\ 0 & \text { otherwise }\end{cases}
$$

Also, $c_{\left(n_{1}, \ldots, n_{r}, \beta\right)}(v Q)=v c_{\left(n_{1}, \ldots, n_{r}, \beta\right)}(Q)=v c_{\beta}\left(Q_{n_{1}, \ldots, n_{r}}\right)=c_{\beta}\left(v Q_{n_{1}, \ldots, n_{r}}\right)$ for every $|\beta|=m-N$.

It is easily seen that mon $\mathscr{P}_{v}\left({ }^{m} E, Y\right) \subseteq$ mon $\mathscr{P}_{v}\left({ }^{m-1} E, Y\right)$; hence $\omega \in$ mon $\mathscr{P}_{v}\left({ }^{m} E, Y\right) \subseteq$ mon $\mathscr{P}_{v}\left({ }^{m-N} E, Y\right)$ for all $1 \leq n \leq N$ and

$$
\begin{aligned}
& \sum_{|\alpha|=m}\left\|c_{\alpha}(P)\right\|\left|z^{\alpha}\right| \\
& \leq \sum_{N=0}^{m} \sum_{n_{1}+\cdots+n_{r}=N} \sum_{|\beta|=m-N}\left\|c_{\left(n_{1}, \ldots, n_{r}, \beta\right)}(P)\right\|\left|z_{1}\right|^{n_{1}} \cdots\left|z_{r}\right|^{n_{r}}\left|\omega_{r+1}\right|^{\beta_{1}}\left|\omega_{r+2}\right|^{\beta_{2}} \ldots \\
& \leq \sum_{N=0}^{m} \sum_{n_{1}+\cdots+n_{r}=N} \sum_{|\beta|=m-N}\left\|c_{\left(n_{1}, \ldots, n_{r}, \beta\right)}(v Q)\right\|\|z\|_{\infty}^{N}\left|\omega_{r+1}\right|^{\beta_{1}}\left|\omega_{r+2}\right|^{\beta_{2}} \ldots \\
&=\sum_{N=0}^{m} \sum_{n_{1}+\cdots+n_{r}=N} \sum_{|\beta|=m-N}\left\|c_{(0, \ldots, 0, \beta)}\left(v Q_{n_{1}, \ldots, n_{r}}\right)\right\|\|z\|_{\infty}^{N}\left|\omega_{r+1}\right|^{\beta_{1}}\left|\omega_{r+2}\right|^{\beta_{2}} \ldots \\
&\left.\quad=\sum_{N=0}^{m}\|z\|_{\infty}^{N} \sum_{n_{1}+\cdots+n_{r}=N} \sum_{|\alpha|=m-N}\left\|c_{\alpha}\left(v Q_{n_{1}, \ldots, n_{r}}\right)\right\||\omega|^{\alpha}\right)<\infty
\end{aligned}
$$

The last expression is finite since each $\sum_{|\alpha|=m-N}\left\|c_{\alpha}\left(v Q_{n_{1}, \ldots, n_{r}}\right)\right\||\omega|^{\alpha}$ is finite because $\omega \in$ mon $\mathscr{P}\left({ }^{m-N} E, Y\right)$ and we then have finite sums of real numbers. This completes the proof.

Let $\left(a_{n}\right)_{n} \subseteq X$ be such that $a_{p^{\alpha}}=0$ whenever $|\alpha| \neq m$. We know from $[6$, Corollary 2] that

$$
\sigma_{u}=\inf \left\{\mu \in \mathbb{R}: \text { there exists } f \in H_{\infty}\left(B_{\ell_{\infty}}, X\right), c_{\alpha}(f)=\frac{a_{p^{\alpha}}}{p^{\mu \alpha}}\right\}
$$

Now, if $f$ is any such function, then we have that $c_{\alpha}(f)=0$ if $|\alpha| \neq m$. Then $f$ is an $m$-homogeneous polynomial and

$$
\sigma_{u}=\inf \left\{\mu \in \mathbb{R}: \text { there exists } Q \in \mathscr{P}\left({ }^{m} \ell_{\infty}, X\right), c_{\alpha}(Q)=\frac{a_{p^{\alpha}}}{p^{\mu \alpha}}\right\}
$$

With this we can give the Proof of Theorem 4.1. In order to keep the notation as simple as possible we write $S=M\left(\mathscr{P}_{v}\left({ }^{m} \ell_{\infty}, Y\right)\right)$. Let us show first that $T_{m}(v) \leq$ $1 / S$. Let us take an $m$-homogeneous Dirichlet polynomial $\sum_{|\alpha|=m} a_{p^{\alpha}} /\left(p^{\alpha}\right)^{s}$ and let $\sigma_{u}$ and $\sigma_{a}$ be the corresponding abscissas of uniform and absolute convergence. Fix $\delta>0$ and let us show that

$$
\sum_{|\alpha|=m} \frac{\left\|v a_{p^{\alpha}}\right\|}{\left(p^{\alpha}\right)^{\sigma_{u}+\frac{1}{S}+\delta}}<\infty
$$


By (18) we can choose $Q \in \mathscr{P}\left({ }^{m} \ell_{\infty}, X\right)$ such that $c_{\alpha}(Q)=\frac{a_{p^{\alpha}}}{\left(p^{\alpha}\right)^{\sigma_{u}+\delta / 3}}$. Then

$$
\sum_{|\alpha|=m} \frac{v a_{p^{\alpha}}}{\left(p^{\alpha}\right)^{\sigma_{u}+\delta / 3}} z^{\alpha} \in \mathscr{P}_{v}\left(\ell_{\infty}, Y\right) .
$$

Let now $r=\frac{1}{S}+\frac{2 \delta}{3}$ and $q=\left(\frac{1}{S}+\frac{\delta}{3}\right)^{-1}$. Then $r q>1$ and $\left(\frac{1}{p_{n}^{r}}\right)_{n} \in \ell_{q} \cap B_{\ell_{\infty}}$, where $\left(p_{n}\right)_{n}$ denotes the sequence of prime numbers.

On the other hand $q<S$; by the very definition of $M\left(\mathscr{P}_{v}\left({ }^{m} \ell_{\infty}, Y\right)\right)$ this implies that $\ell_{q} \subseteq$ mon $\mathscr{P}_{v}\left({ }^{m} \ell_{\infty}, Y\right)$. Then

$$
\begin{aligned}
\sum_{|\alpha|=m} \frac{\left\|v a_{p^{\alpha}}\right\|}{\left(p^{\alpha}\right)^{\sigma_{u}+\frac{1}{S}+\delta}}=\sum_{|\alpha|=m} & \frac{\left\|v a_{p^{\alpha}}\right\|}{\left(p^{\alpha}\right)^{\sigma_{u}+\frac{\delta}{3}+\frac{1}{S}+\frac{2 \delta}{3}}} \\
& =\sum_{|\alpha|=m} \frac{\left\|v a_{p^{\alpha}}\right\|}{\left(p^{\alpha}\right)^{\sigma_{u}+\frac{\delta}{3}}}\left(\frac{1}{p^{r}}\right)^{\alpha}=\sum_{|\alpha|=m}\left\|c_{\alpha}(v Q)\left(\frac{1}{p_{n}}\right)^{\alpha}\right\|<\infty ;
\end{aligned}
$$

and we have $T_{m}(v) \leq 1 / S$. In order to prove the converse inequality let us begin by fixing $0<\delta<1 / S$ and defining $q=\left(\frac{1}{S}+\frac{\delta}{2}\right)^{-1}>S$. Let $\varepsilon=\left(\varepsilon_{n}\right)_{n} \in \ell_{q} \cap B_{\ell_{\infty}}$ such that $\varepsilon \notin$ mon $\mathscr{P}_{v}\left({ }^{m} \ell_{\infty}, Y\right)$. Let us note that if $z=\left(z_{n}\right)_{n} \in \operatorname{mon} \mathscr{P}_{v}\left({ }^{m} \ell_{\infty}, Y\right)$, then for every bijective $\sigma: \mathbb{N} \rightarrow \mathbb{N}$ we have $\left(z_{\sigma(n)}\right)_{n} \in$ mon $\mathscr{P}_{v}\left({ }^{m} \ell_{\infty}, Y\right.$ ) (see e.g. the argument given in $\left[6\right.$, page 550]). Hence we can assume that $\varepsilon_{n}$ is non-increasing and then that $\left(n^{1 / q} \varepsilon_{n}\right)_{n}$ is bounded. By the prime number theorem there exists $K>0$ such that $p_{n} \leq K n \log n$ for every $n \geq 2$. Then let us define $\eta_{n}=\left(p_{n}^{\frac{1}{S}-\delta}\right)^{-1}$ and we have

$$
\begin{aligned}
0<\frac{\varepsilon_{n}}{\eta_{n}}=\varepsilon_{n} p_{n}^{\frac{1}{S}-\delta}=\varepsilon_{n} n^{1 / q} \frac{p_{n}^{\frac{1}{S}-\delta}}{n^{1 / q}}= & \varepsilon_{n} n^{1 / q} \frac{p_{n}^{\frac{1}{S}-\delta}}{n^{\frac{1}{S}-\frac{\delta}{2}}} \\
& =\varepsilon_{n} n^{1 / q}\left(\frac{p_{n}}{n}\right)^{\frac{1}{S}-\delta} \frac{1}{n^{\frac{\delta}{2}}} \leq \varepsilon_{n} n^{1 / q} \frac{(K \log n)^{\frac{1}{S}-\delta}}{n^{\frac{\delta}{2}}} .
\end{aligned}
$$

The last sequence tends to 0 , hence there exists $n_{0}$ so that $\varepsilon_{n} \leq \eta_{n}$ for every $n \geq n_{0}$ and this implies by Lemma 4.2 that $\eta \notin$ mon $\mathscr{P}_{v}\left({ }^{m} \ell_{\infty}, Y\right)$. This means that there exists $P \in \mathscr{P}_{v}\left({ }^{m} \ell_{\infty}, Y\right)$ such that $\sum_{|\alpha|=m}\left\|c_{\alpha}(P)\right\||\eta|^{\alpha}=\infty$. Let us write $P=v Q$ and $a_{p^{\alpha}}=c_{\alpha}(Q)$. Since $\sum_{|\alpha|=m} \frac{c_{\alpha}(Q)}{p^{0 \alpha}} z^{\alpha}$ is the monomial series expansion of $Q \in \mathscr{P}\left({ }^{m} \ell_{\infty}, X\right)$ we have that the series $\sum_{|\alpha|=m} \frac{a_{p^{\alpha}}}{\left(p^{\alpha}\right)^{s}}$ has abscissa of uniform convergence $\sigma_{u} \leq 0$. Hence $\sigma_{a}-\sigma_{u} \geq \sigma_{a}$. On the other hand

$$
\sum_{|\alpha|=m} \frac{\left\|v a_{p^{\alpha}}\right\|}{\left(p^{\alpha}\right)^{\frac{1}{S}-\delta}}=\sum_{|\alpha|=m}\left\|v c_{\alpha}(Q)\right\||\eta|^{\alpha}=\infty .
$$

Therefore $\sigma_{a}-\sigma_{u} \geq \sigma_{a} \geq \frac{1}{S}-\delta$; this gives that $T_{m}(v) \geq \frac{1}{S}-\delta$ for all $\delta$ and finally $T_{m}(v) \geq \frac{1}{S}$.

\section{REFERENCES}

[1] G. Bennett. Inclusion mappings between $l^{p}$ spaces. J. Functional Analysis, 13:20-27, 1973. 
[2] H. F. Bohnenblust and E. Hille. On the absolute convergence of Dirichlet series. Ann. of Math. (2), 32(3):600-622, 1931.

[3] H. Bohr. Über die Bedeutung der Potenzreihen unendlich vieler Variabeln in der Theorie der Dirichlet-schen Reihen $\sum \frac{a_{n}}{n^{2}}$. Nachr. Ges. Wiss. Göttingen, Math. Phys. Kl., pages 441-488, 1913.

[4] H. Bohr. Über die gleichmäßige Konvergenz Dirichletscher Reihen. J. Reine Angew. Math., 143:203-211, 1913.

[5] B. Carl. Absolut- $(p, 1)$-summierende identische Operatoren von $l_{u}$ in $l_{v}$. Math. Nachr., 63:353360, 1974.

[6] A. Defant, D. García, M. Maestre, and D. Pérez-García. Bohr's strip for vector valued Dirichlet series. Math. Ann., 342(3):533-555, 2008.

[7] A. Defant, M. Maestre, and C. Prengel. The arithmetic Bohr radius. Q. J. Math., 59(2):189$205,2008$.

[8] A. Defant, M. Maestre, and C. Prengel. Domains of convergence for monomial expansions of holomorphic functions in infinitely many variables. J. Reine Angew. Math., 634:13-49, 2009.

[9] A. Defant and P. Sevilla-Peris. Convergence of Dirichlet polynomials in Banach spaces. Trans. Amer. Math. Soc., to appear, 2009.

[10] A. Defant and P. Sevilla-Peris. A new multilinear insight on the classical Littlewood's $4 / 3$ inequality. J. Funct. Anal., 256(5):1642-1664, 2009.

[11] A. Defant and P. Sevilla-Peris. Convergence of Dirichlet polynomials in Banach spaces. Trans. Amer. Math. Soc., to appear, 2011.

[12] J. Diestel, H. Jarchow, and A. Tonge. Absolutely summing operators, volume 43 of Cambridge Studies in Advanced Mathematics. Cambridge University Press, Cambridge, 1995.

[13] S. Dineen. Complex analysis on infinite-dimensional spaces. Springer Monographs in Mathematics. Springer-Verlag London Ltd., London, 1999.

[14] L. Lempert. The Dolbeault complex in infinite dimensions. II. J. Amer. Math. Soc., 12(3):775793, 1999.

[15] R. A. Ryan. Holomorphic mappings on $l_{1}$. Trans. Amer. Math. Soc., 302(2):797-811, 1987.

[16] N. Tomczak-Jaegermann. Banach-Mazur distances and finite-dimensional operator ideals, volume 38 of Pitman Monographs and Surveys in Pure and Applied Mathematics. Longman Scientific \& Technical, Harlow, 1989.

\section{REFERENCES}

[1] G. Bennett. Inclusion mappings between $l^{p}$ spaces. J. Functional Analysis, 13:20-27, 1973.

[2] H. F. Bohnenblust and E. Hille. On the absolute convergence of Dirichlet series. Ann. of Math. (2), 32(3):600-622, 1931.

[3] H. Bohr. Über die Bedeutung der Potenzreihen unendlich vieler Variabeln in der Theorie der Dirichlet-schen Reihen $\sum \frac{a_{n}}{n^{2}}$. Nachr. Ges. Wiss. Göttingen, Math. Phys. Kl., pages 441-488, 1913.

[4] H. Bohr. Über die gleichmäßige Konvergenz Dirichletscher Reihen. J. Reine Angew. Math., 143:203-211, 1913.

[5] B. Carl. Absolut- $(p, 1)$-summierende identische Operatoren von $l_{u}$ in $l_{v}$. Math. Nachr., 63:353360, 1974

[6] A. Defant, D. García, M. Maestre, and D. Pérez-García. Bohr's strip for vector valued Dirichlet series. Math. Ann., 342(3):533-555, 2008.

[7] A. Defant, M. Maestre, and C. Prengel. The arithmetic Bohr radius. Q. J. Math., 59(2):189205, 2008.

[8] A. Defant, M. Maestre, and C. Prengel. Domains of convergence for monomial expansions of holomorphic functions in infinitely many variables. J. Reine Angew. Math., to appear, 2009.

[9] A. Defant and P. Sevilla-Peris. Convergence of Dirichlet polynomials in Banach spaces. Trans. Amer. Math. Soc., to appear, 2011.

[10] A. Defant and P. Sevilla-Peris. A new multilinear insight on the classical Littlewood's $4 / 3$ inequality. J. Funct. Anal., 256(5):1642-1664, 2009.

[11] J. Diestel, H. Jarchow, and A. Tonge. Absolutely summing operators, volume 43 of Cambridge Studies in Advanced Mathematics. Cambridge University Press, Cambridge, 1995.

[12] S. Dineen. Complex analysis on infinite-dimensional spaces. Springer Monographs in Mathematics. Springer-Verlag London Ltd., London, 1999.

[13] L. Lempert. The Dolbeault complex in infinite dimensions. II. J. Amer. Math. Soc., 12(3):775793,1999 . 
[14] R. A. Ryan. Holomorphic mappings on $l_{1}$. Trans. Amer. Math. Soc., 302(2):797-811, 1987.

[15] N. Tomczak-Jaegermann. Banach-Mazur distances and finite-dimensional operator ideals, volume 38 of Pitman Monographs and Surveys in Pure and Applied Mathematics. Longman Scientific \& Technical, Harlow, 1989.

Institute of mathematics, Carl von Ossietzky University, D-26111 Oldenburg, GerMANY

E-mail address: defant@mathematik.uni-oldenburg.de

Instituto Universitario de Matemática Pura y Aplicada and DMA, ETSiAmn, Edificio 3P, Universidad Politécnica de Valencia, E-46010 Valencia, Spain \& Institute of mathematics, Carl von Ossietzky University, D-26111 Oldenburg, Germany

E-mail address: psevilla@mat.upv.es 